\title{
Investigation of Troponin Use in Inpatient Wards: Real-Life Data
}

\author{
Onur Akhan* and Isa Ardahanli \\ Bilecik Training and Research Hospital, Cardiology Department, Bilecik, Turkey \\ *Corresponding author: Onur Akhan, Bilecik Training and Research Hospital, Cardiology Department, Bilecik, Turkey
}

\begin{tabular}{ll}
\hline ARTICLE INFO & ABSTRACT \\
\hline
\end{tabular}

Received: 幽 May 03, 2021

Published: May 12, 2021

Citation: Onur Akhan, Isa Ardahanli. Investigation of Troponin Use in Inpatient Wards: Real-Life Data. Biomed J Sci \& Tech Res 35(5)-2021. BJSTR. MS.ID.005753.

Keywords: Troponin; Myocardial Damage; Diagnostic Codes; Inpatient Wards

Abbreviations: ICD: Classification of Diseases; Dis: Disease; Obst: Obstructive; Refl: Reflux; Inf: Infection
Introduction: Cardiac troponins are biomarkers which used to show myocardial damage. In some situations, even if the clinical findings do not overlap, it can be used for diagnostic exclusion. Our study aims to evaluate the use and suitability of troponin in inpatient services.

Methods: 1322 patients aged 18 years and older who had at least one troponin evaluation during their hospitalization in 2019 were included in this retrospective study. The patients were then divided into five groups according to their departments. Age and gender information and troponin related data (total tests, follow-up, diagnosis codes) were evaluated.

Results: A total of 1560 troponin evaluations were made, and the highest number of patients were followed in chest diseases (49\%), internal diseases (36.4\%), and cardiology departments $(9.4 \%)$. Control troponin follow-up is most frequently observed in anesthesiology (40.6\%). Highest rate of abnormal detection of first and control troponin values are anesthesiology (first:75\%, control:82.9\%) and cardiology (first:26.6\%, control:85.7\%), the lowest rate of abnormal detection of the first troponin value were internal diseases $(9.1 \%)$ and chest diseases (11.4\%). The most common diagnostic codes are dyspnea (15.6\%), chest pain (14.2\%), and hypertension (9.7\%), differences are observed on departments.

Conclusion: In light of our study's findings, we can state that troponin assessment is frequently used in inpatient wards and some cases inappropriately. Abnormal result rates differ based on departments, trainings can be planned, and the efficiency of the existing rotation training can be increased to ensure standardization for the relevant inspection. The adverse outcomes (unnecessary evaluation, increased anxiety) and financial resources are potential adverse outcomes in this situation.

\section{Introduction}

Cardiac troponins are components of the part that cause myocardial cells to contract, and almost all of them are found in the heart $[1,2]$. An increase above 99 percent of the upper reference limit of at least one of the cardiac troponin evaluations is defined as myocardial damage [1-4]. Increases can be seen other than ischemic heart disease, which is not as rare as expected [5,6]. The main non-ischemic mechanisms in cardiac troponin increase can be listed as mechanical stress due to preload increase, hypoxemia, hypotension, shock table, kidney diseases, anemia, ventricular arrhythmias, and heart failure. Sepsis, infectious diseases, stroke, subarachnoid hemorrhage, pulmonary embolism, pulmonary hypertension, infiltrative diseases, chemotherapeutic agents, critically ill patients, physiological stress, and intense exercise can be listed among other systemic causes that can cause troponin increase [7-10]. The reason for cardiac troponin increase due to multifactorial factors may not always be differentiated [4].

Troponin evaluations in hospitals, especially in emergency wards, can be performed inappropriately as a routine examination in some cases, for example, in elderly patients, with the thought of exclusion and harmless, even if acute coronary syndrome is 
not suspected [11,12]. Inappropriate pathology test is defined as tests that can be avoided without causing pathological results in patient care [13]. In the 2020 NSTEMI (non-ST elevation myocardial infarction) guideline, follow-up with high-sensitivity troponin is recommended. The threshold values specific to the high-sensitive troponins are recommended and with $0 \mathrm{~h}-1 \mathrm{~h}, 0 \mathrm{~h}-2 \mathrm{~h}$, and $0 \mathrm{~h}-3 \mathrm{~h}$ procedures $[14,15]$. High-sensitivity cardiac troponin I and troponin $\mathrm{T}$ are recommended markers in current guidelines for use in myocardial damage [4,14]. Although inappropriate troponin evaluations are thought to be harmless, in some cases, they can cause overdiagnosis and treatment, which may cause unnecessary treatment to the patient and even harm the patient. These evaluations can increase the rate of false positivity and cause an increase in anxiety in patients. Due to the increase in false positivity rates, the repetition of the examinations, and the initiation of a process that is unnecessary for further examinations [12,16-18]. Inadequate use of the hospital's financial resources and the inability to direct the resources are other possible negativities. It has been shown that the annual cost of the troponin assessment can be as high as $\$ 115,000[19,20]$.

Studies in the literature on inappropriate troponin use are mostly related to emergency wards $[12,19,20]$. Troponin evaluations can also be made in hospital inpatient wards in cases of cardiac suspicion and sometimes routinely. In addition to the negativities mentioned above, it can be predicted that it may cause an increased consultation burden with the relevant troponin evaluations' effect without any cardiological requirement. When the literature is reviewed, troponin evaluation was made in $29 \%$ of inpatients in a study conducted on troponin evaluation in inpatients. No chest pain or ischemic heart disease diagnosis was found in $89 \%$ of patients evaluated for troponin. In this study, no evaluation was made on a department basis [21]. Our study aimed to examine troponin evaluations made in inpatient wards of our hospital on a department basis to reveal the findings and investigate their appropriateness. Thus, in the future, under the guidance of real-life data of our hospital, it can be planned to make arrangements with trainings on troponin use.

\section{Methods}

Our study is a retrospective cohort study. A total of 1322 patients treated in inpatient wards in 2019 and had troponin evaluation at least once during their hospitalization were included.

Table 1: Basic characteristics and troponin evaluation.
Patients under the age of 18 in the pediatric age group were not included in the study. These patients were later divided into five separate groups: cardiology, chest diseases, internal medicine, anesthesiology (6-bed intensive care unit where non-intubated patients were monitored), and other departments (neurology, orthopedics, physical therapy and rehabilitation, general surgery, etc.). Age and gender data of the patients were obtained in terms of demographic data. Data about the patients were evaluated using the hospital information system with the approval of the institution. In our hospital, troponin evaluations are made in the biochemistry laboratory with the high-sensitivity troponin I of the "Abbott, Architect" brand. Control troponin evaluations of the patients were also included in the study. In our study, troponin's upper reference value was accepted as the reference value of our hospital's laboratory as $17.5 \mathrm{pg} / \mathrm{ml}$. Above this value was stated as abnormal (high) troponin value. In the study, the patients' diagnoses were determined according to the ICD-10 (Classification of diseases) codes in the hospital information system [22]. Basic descriptive statistical methods, including mean, percentage, and standard deviation, have been used to reveal the available data's preliminary evaluation.

\section{Results}

After categorizing the patients included in our study according to the departments of science in which they were followed, the first three departments with the highest number of patients were ranked as chest diseases, internal diseases, and cardiology departments, respectively (49\%,36.4\%, and 9.4\%, respectively). The study's basic characteristics such as age, gender, and troponin evaluations are shown in Table 1. In our study, a total of 1560 troponin measurements were evaluated, including control troponin assessments. The control troponin evaluation rate and the abnormal initial troponin evaluation rate were highest in the anesthesiology department (40.6\%,75\%, respectively). The rate of detection of high control troponins is mostly in cardiology (85.7\%), followed by anesthesiology (82.4\%) and internal diseases (58.2\%), respectively. The departments with the lowest abnormal initial troponin values are internal diseases (9.1\%) and chest diseases (11.4\%), respectively. The most common diagnoses in the whole population were dyspnea, chest pain, and hypertension. The most frequent diagnoses entered based on departments are also given in Table 2.

\begin{tabular}{|c|c|c|c|c|c|c|}
\hline & Cardiology & Chest Diseases & $\begin{array}{c}\text { Internal } \\
\text { Medicine }\end{array}$ & $\begin{array}{c}\text { Anaesthesiology } \\
\text { Intensive care }\end{array}$ & Other Sciences & Total \\
\hline Patient, n, & 124 & 648 & 482 & 32 & 36 & 1322 \\
\hline$\%$ & $9.4 \%$ & $49 \%$ & $36.4 \%$ & $2.5 \%$ & $2.7 \%$ & $100 \%$ \\
\hline Gender (male) n, & 73 & 369 & 202 & 18 & 9 & 671 \\
\hline$\%$ & $58.8 \%$ & $56.9 \%$ & $41.9 \%$ & $56.2 \%$ & $25 \%$ & $50.7 \%$ \\
\hline Age, & 60.0 & 59.2 & 63.3 & 74.2 & 62.8 & 61.1 \\
\hline
\end{tabular}




\begin{tabular}{|c|c|c|c|c|c|c|c|}
\hline \multicolumn{2}{|c|}{ mean (SD) } & $(16.6)$ & $(17.2)$ & $(14.8)$ & $(10.2)$ & $(19.9)$ & $(16.5)$ \\
\hline \multicolumn{8}{|c|}{ Troponin Measurements } \\
\hline \multicolumn{2}{|c|}{ Patient n, \% } & 112 & 578 & 459 & 19 & 28 & 1196 \\
\hline \multicolumn{2}{|c|}{ (1 measurement) } & $90.3 \%$ & $89.1 \%$ & $95.2 \%$ & $59.4 \%$ & $77.8 \%$ & $90.5 \%$ \\
\hline \multicolumn{2}{|c|}{ Patient n, \% } & 12 & 70 & 23 & 13 & 8 & 126 \\
\hline \multicolumn{2}{|c|}{$(2 \leq$ measurement $)$} & $9.7 \%$ & $10.9 \%$ & $4.8 \%$ & $40.6 \%$ & $22.2 \%$ & $9.5 \%$ \\
\hline \multirow{4}{*}{$\begin{array}{c}\text { Abnor. } \\
\text { Value n, \% }\end{array}$} & \multirow{2}{*}{$* 1^{\text {st }}$} & 33 & 74 & 44 & 24 & 7 & 182 \\
\hline & & $26.6 \%$ & $11.4 \%$ & $9.1 \%$ & $75.0 \%$ & $19.4 \%$ & $13.7 \%$ \\
\hline & $* * 2^{\text {nd }}$ & $18 / 21$ & $40 / 109$ & $32 / 55$ & $34 / 41$ & $6 / 12$ & $130 / 238$ \\
\hline & $\ldots$ & $85.7 \%$ & $36.6 \%$ & $58.2 \%$ & $82.9 \%$ & $50 \%$ & $54.6 \%$ \\
\hline \multirow{2}{*}{\multicolumn{2}{|c|}{ All measurements }} & 145 & 757 & 537 & 73 & 48 & 1560 \\
\hline & & $9.2 \%$ & $48.6 \%$ & $34.4 \%$ & $4.7 \%$ & $3.1 \%$ & $100 \%$ \\
\hline
\end{tabular}

Note: n: number, SD: standard deviation, trop: troponin, abnor.: abnormal (trop $>17.5$ pg/ml),

*:first measurement, **:second and subsequent measurements

Table 2: Common ICD codes and chief complaints of patients.

\begin{tabular}{|c|c|c|}
\hline & ICD Codes/Diagnoses & All Patients - ICD Codes/Complaints \\
\hline \multirow{4}{*}{ Cardiology } & 1. Chest Pain $(33.9 \%)$ & Dyspnea $(15.6 \%)$ \\
\hline & 2. Coronary Artery Dis. (24.2\%) & Chest Pain (14.2\%) \\
\hline & 3. Hypertension $(11.3 \%)$ & Hypertension $(9.7 \%)$ \\
\hline & 4. Dyspnea $(10.5 \%)$ & Chronic Obst. Lung Dis. (6.2\%) \\
\hline \multirow{4}{*}{ Chest Diseases } & 1. Dyspnea $(27.3 \%)$ & Cough $(5.7 \%)$ \\
\hline & 2. Chest Pain $(21.3 \%)$ & Gastroesophageal Reflux Dis. (5.2\%) \\
\hline & 3. Chronic Obst. Lung Dis. (12.0\%) & Asthma/Allergy (3.4\%) \\
\hline & 4. Cough $(11.6 \%)$ & Upper Respiratory Tract Inf. (2.7\%) \\
\hline \multirow{4}{*}{ Internal Medicine } & 1. Hypertension $(23.9 \%)$ & Pneumonia $(2.6 \%)$ \\
\hline & 2. General Medical Treat. (18.5\%) & Myalgia (2.6\%) \\
\hline & 3. Gastroesophageal Refl. Dis. (14.5\%) & Coronary Artery Dis. (2.3\%) \\
\hline & 4. Hormonal Dis. (10.9\%) & Renal Deficiency (1.7\%) \\
\hline \multirow{4}{*}{$\begin{array}{l}\text { Anaesthesiology } \\
\text { Intensive care }\end{array}$} & 1. Dyspnea $(40.6 \%)$ & Anemia $(1.3 \%)$ \\
\hline & 2. Upper Respiratory Tract Inf. (12.5\%) & Irritable Bowel Syndrome (1.2\%) \\
\hline & 3. Nausea/Vomiting (9.3\%) & General Weakness (1.0\%) \\
\hline & 4. Serebrovascular Events (\%6.3) & Extremity Disorders (0.9\%) \\
\hline \multirow{4}{*}{$\begin{array}{l}\text { Other } \\
\text { Sciences }\end{array}$} & 1. Extremity Disorders (33.3\%) & Vitamin Deficiency (0.9\%) \\
\hline & 2. Abdominal Pain (11.1\%) & Congestive Heart Failure (0.9\%) \\
\hline & 3. General Weakness (8.3\%) & Cerebrovascular Events (0.9\%) \\
\hline & 4. Serebrovascular Events (8.3\%) & $\ldots$ \\
\hline
\end{tabular}

\section{Discussion}

Troponin is a widely used marker to detect myocardial damage in suspected acute coronary syndrome [1-4]. In cases where clinical findings are insufficient and clinical information cannot be obtained from the patient entirely, troponin evaluation can be performed for diagnostic exclusion purposes and sometimes routinely [11,12]. Troponin assessment and follow-up are most frequently used in emergency services to diagnose acute coronary syndrome and monitor these patients in cardiology services. Troponin use is of great importance, especially in centers where invasive cardiological interventions are performed [2,12,14,15]. Therefore, it can be predicted that the most frequent troponin use outside the emergency room should be in cardiology services. Our study aimed to reveal the findings by troponin evaluations based on departments in patients hospitalized in our hospital wards and examine troponin evaluations' appropriateness.

In our study, the departments for which troponin was evaluated most frequently are listed as chest diseases (49\%), internal diseases (36.4\%), and cardiology units (9.4\%). The patient population associated with chest diseases in our province 
is relatively higher due to the location of the province and the existing factories; there are relatively few patients who underwent troponin follow-up due to not performing invasive cardiological interventions in our hospital, and if necessary, referral of these patients to advanced centers and follow-up there. The frequency of patients who were evaluated for troponin due to these conditions of our city and hospital may have been encountered in this way. Considering the rate of troponin evaluation in the study population of the patients who were followed up with control troponin on a branch basis, it was observed that the control troponin follow-up was most common in the group of anesthesiology intensive care (40.6\%) and other departments group (22.2\%). The high rate of high initial troponin measurements is also seen in patients under anesthesiology (75.0\%).

The follow-up of the oldest population, the patients in critical condition requiring monitoring and patients due to acute coronary syndrome who are not referring to a center where invasive intervention can be performed may cause these results (Total population mean age 61.1, anesthesiology mean age 74.2). The second department in which the high rate of detection of the first troponin evaluation is detected most frequently is cardiology (26.6\%). High control troponin measurements were mostly in cardiology (85.7\%) and anesthesiology (82.9\%) departments. It can be stated that the inclusion of the diagnosis and follow-up of the related diseases in the specialty training might affect in determining the high rates in the cardiology department. The lowest rate of high detection of the first troponin evaluations was found in internal medicine departments (9.1\%) and chest diseases (11.4\%).

Frequent follow-up of possible secondary pathologies that may cause inappropriate troponin elevation in the internal medicine department. In the chest diseases department, frequent follow-up of diseases associated with dyspnea is one of the most prominent angina equivalents that may cause inappropriate troponin evaluations. When the evaluation of the diagnosis codes, it is seen that the most common diagnosis-related ICD codes in the whole population are dyspnea, chest pain, and hypertension. Besides, dyspnea is among the most common reasons based on departments. However, considering the current numbers and rates, even though there are cardiology rotations in the two branches of specialization training, the subject's training can be given during the specialization process. Thus, the use of the relevant examinations over the necessity can be prevented.

Also, arrangements can be made for rotation training. A study conducted showed that the use of inappropriate tests decreased from $43 \%$ to $22 \%$ after a training process that included acute coronary syndrome diagnosis criteria and potential clinical symptoms and questionnaire evaluation [23]. In 2014, in line with the information obtained from the information system of 14 hospitals, it was seen that approximately $29 \%$ of 130 thousand inpatients had troponin evaluation. It was observed that the most common diagnoses of these patients were sepsis and pneumonia, and only $11 \%$ of the patients who were evaluated for troponin were diagnosed with ischemic heart diseases and chest pain [21]. When we look at the findings and results of our study with diagnostic codes, it can be stated that there is no standardization in the information entered into patients. In the patients' diagnostic codes, there are different pieces of information, including the names of the diseases, some of which include symptoms. In addition, it was observed that some patients were entered with a general medical treatment code without any pre-diagnosis. Accompanied by these findings, it is necessary to provide a standardization on this subject and again, this situation should be explained to all branches in the training processes.

Our study data could not be compared in terms of other findings since no detailed study could be reached other than the study conducted by Farber et al. on troponin evaluation on hospitalized patients other than emergency departments in the literature [21]. In our study, the time between troponin follow-ups and troponin change rates could not be evaluated. In future studies, multi-center studies in which these data will be examined in detail with more patients. The outcome includes the rate of detection of acute coronary syndrome, other cardiac outcomes, cardiology consultations, and patient anxiety conditions will also be evaluated. Thus, by enabling troponin evaluations to be used more effectively in the light of guidelines, inappropriate test-related adverse outcomes can be prevented. We can state that our study will contribute to the literature, with the evaluations and findings based on the departments, and that it may be predictive for future studies.

\section{Limitations}

In our study, intubated patients were not evaluated. Therefore, the findings related to the anesthesiology department may not fully meet the target population. Due to the absence of a coronary angiography unit in our hospital, patients diagnosed with acute coronary syndrome are not followed up in our hospital. The referral process to the center in which invasive procedures can be made begins in the case of such a condition in hospitalized patients. Therefore, we can state that our study cannot be generalized to cardiology clinics in which invasive cardiac procedures are also performed.

\section{Conclusion}

As a result, troponin evaluation is frequently used in inpatient services, and the abnormal results differ with departments. Although there are cardiology guideline data on the subject, there are different approaches between departments. Training seminars can be organized in hospitals with all departments' participation to ensure this situation's standardization. Thus, the workload will be distributed appropriately, and financial resources will be used more appropriately. 


\section{Acknowledgment and Funding}

All authors declare that no conflict of interest and no financial support.

\section{Author Contribution Form}

Authors' contributions to the study: OA: Planning the study, collecting data, data analysis, and evaluation. İA: Data collection, data analysis, and evaluation.

\section{References}

1. Thygesen K, Mair J, Katus H (2010) The Study Group on Biomarkers in Cardiology of the ESC Working Group on Acute Cardiac Care. Recommendations for the use of cardiac troponin measurement in acute cardiac care. Eur Heart J 31(18): 2197-2204.

2. Thygesen K, Mair J, Giannitsis E (2012) Study Group on Biomarkers in Cardiology of the ESC Working Group on Acute Cardiac Care. How to use high-sensitivity cardiac troponins in acute cardiac care. Eur Heart J 33 2252-2257

3. Apple FS, Jaffe AS, Collinson P (2015) On behalf of the International Federation of Clinical Chemistry (IFCC) Task Force on Clinical Applications of Cardiac Bio-Markers. IFCC educational materials on selected analytical and clinical applications of high sensitivity cardiac troponin assays. Clin Biochem 48(4-5): 201-203.

4. Thygesen K, Alpert JS, Jaffe AS (2018) Executive Group on behalf of the Joint European Society of Cardiology (ESC)/American College of Cardiology (ACC)/American Heart Association (AHA)/World Heart Federation (WHF) Task Force for the Universal Definition of Myocardial Infarction. Fourth Universal Definition of Myocardial Infarction (2018). Circulation 138(20): e618-e651.

5. Vestergaard KR, Jespersen CB, Arnadottir A (2016) Prevalence and significance of troponin elevations in patients without acute coronary disease. Int J Cardiol 222: 819-825.

6. Schmid J, Liesinger L, Birner Gruenberger R (2018) Elevated cardiac troponin T in skeletal myopathies. J Am Cardiol Coll 71(14): 1540-1549.

7. Weil BR, Suzuki G, Young RF (2018) Troponin release and reversible left ventricular dysfunction following transient pressure overload: Stress induced myocardial stunning. J Am Cardiol Coll 71(25): 2906-2916.

8. Siriwardena M, Campbell V, Richards AM (2012) Cardiac biomarker responses to dobutamine stress echocardiography in healthy volunteers and patients with coronary artery disease. Clin Chem 58(10): 14921494.

9. Eggers KM, Lindahl B (2017) Application of cardiac troponin in cardiovascular diseases other than acute coronary syndrome. Clin Chem 63(1): 223-235.

\section{ISSN: 2574-1241}

DOI: 10.26717/BJSTR.2021.35.005753

Onur Akhan. Biomed J Sci \& Tech Res

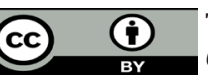

This work is licensed under Creative Commons Attribution 4.0 License

Submission Link: https://biomedres.us/submit-manuscript.php
10. Giannitsis E, Katus HA (2013) Cardiac troponin level elevations not related to acute coronary syndromes. Nat Rev Cardiol 10(11): 623-634.

11. Sandoval Y, Gunsolus IL, Smith SW (2019) Appropriateness of Cardiac Troponin Testing: Insights from the Use of TROPonin in Acute coronary syndromes (UTROPIA) Study. Am J Med 132(7): 869-874.

12. Gardiner FW, Zhai S (2017) Appropriate pathology ordering? Troponin testing within an Australian Emergency Department. Ir J Med Sci 186(1): 213-218.

13. Stuart W, Smellie A (2014) Inappropriate pathology testing; is there really a problem? Chem Pathol 46(1): S13-S15.

14. Collet JP, Thiele H, Barbato E (2020) ESC Scientific Document Group. 2020 ESC Guidelines for the management of acute coronary syndromes in patients presenting without persistent ST-segment elevation. Eur Heart J 42(14): 1289-1367.

15. Roffi M, Patrono C, Collet JP (2016) ESC Scientific Document Group. 2015 ESC Guidelines for the management of acute coronary syndromes in patients presenting without persistent ST-segment elevation: Task Force for the Management of Acute Coronary Syndromes in Patients Presenting without Persistent ST-Segment Elevation of the European Society of Cardiology (ESC). Eur Heart J 37(3): 267315

16. Hammett RJ, Harris RD (2002) Halting the growth in diagnostic testing. Med J Aust 177(3): 124-125.

17. Sah S, Elias P, Ariely D (2013) Investigation momentum: the relentless pursuit to resolve uncertainty. JAMA Intern Med 173(10): 932-933.

18. Morgan S, Coleman J (2014) We live in testing times-teaching rational test ordering in general practice. Aust Fam Physician 43(5): 273-276.

19. Groarke JD, Browne L, Margey R (2013) A multicentre analysis of troponin use in clinical practice. Ir J Med Sci 182(2): 185-190.

20. Riley RF, Miller CD, Russell GB (2017) Cost analysis of the History, ECG, Age, Risk factors, and initial Troponin (HEART) Pathway randomized control trial. Am J Emerg Med 35(1): 77-81.

21. Farber AJ, Suarez K, Slicker K (2017) Frequency of Troponin Testing in Inpatient Versus Outpatient Settings. Am J Cardiol 119(8): 1153-1155.

22. (2010) WHO Library Cataloguing-in-Publication Data International statistical classification of diseases and related health problems $\left(10^{\text {th }}\right.$ Edn.)., p. 3.

23. Scorgie R, Nicholls GM, Jones P (2014) Association between an educational intervention and a reduction in inappropriate troponin testing in patients presenting to an adult emergency department. Intern Med J 44(11): 1100-1108.

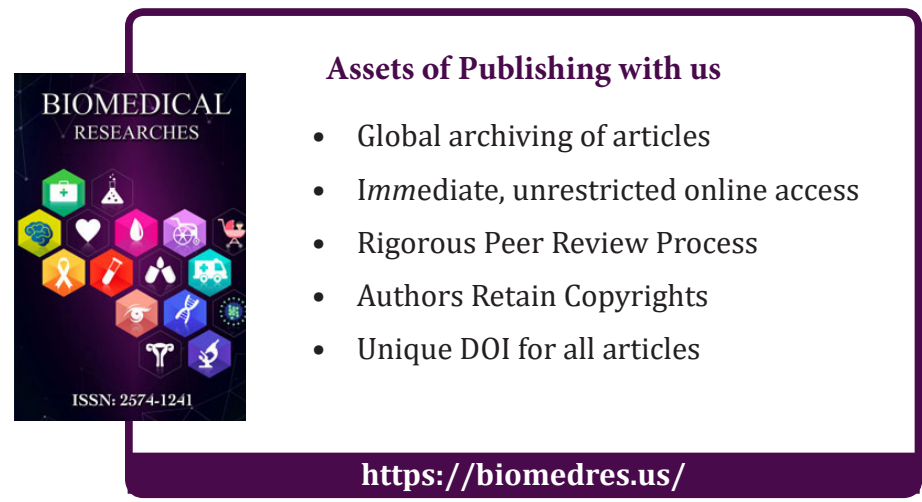

